\title{
RHEUMATOID HAND DEFORMITIES CONSIDERED IN THE LIGHT OF TENDON IMBALANCE. I
}

\author{
O. J. VAUGHAN-JACKSON, London, ENGLAND
}

Until ten or fifteen years ago the problems presented by disabilities of the hand in rheumatoid arthritis were neglected because it was believed that surgery had little to offer: but more recently interest in these rheumatoid affections, as in hand surgery in general, has been awakened in many countries. My own interest was aroused in 1946 by the discovery of two cases of attrition rupture of extensor tendons at the inferior radio-ulnar joint. These tendon ruptures convinced me that far too much in the rheumatoid hand was being accepted as an inevitable consequence of arthritis. The study of rheumatoid deformities proved fascinating. It has thrown new light on the abnormal mechanisms underlying the deformities, and at the same time research into the anatomy and function of the tendons and small muscles of the hand has revealed the shortcomings of standard accounts of rheumatoid deformities.

It may be argued that this is a sterile and limited field, and that the limited gains from surgery are likely to be undone by progress of the disease. Nevertheless, pursuit of this problem will add not only to our knowledge of abnormal anatomy and function in the hand but also to our understanding of the normal, which is still incomplete. Moreover, we may well expect advances in the control of this disease and possibly even its cure. Surgeons must be ready with a knowledge of the mechanical problems involved and of what may be expected from reconstructive surgery. Even without such advance in control of the disease it is still necessary to reassess the part that surgery can take in the treatment of disabilities of the hand in rheumatoid

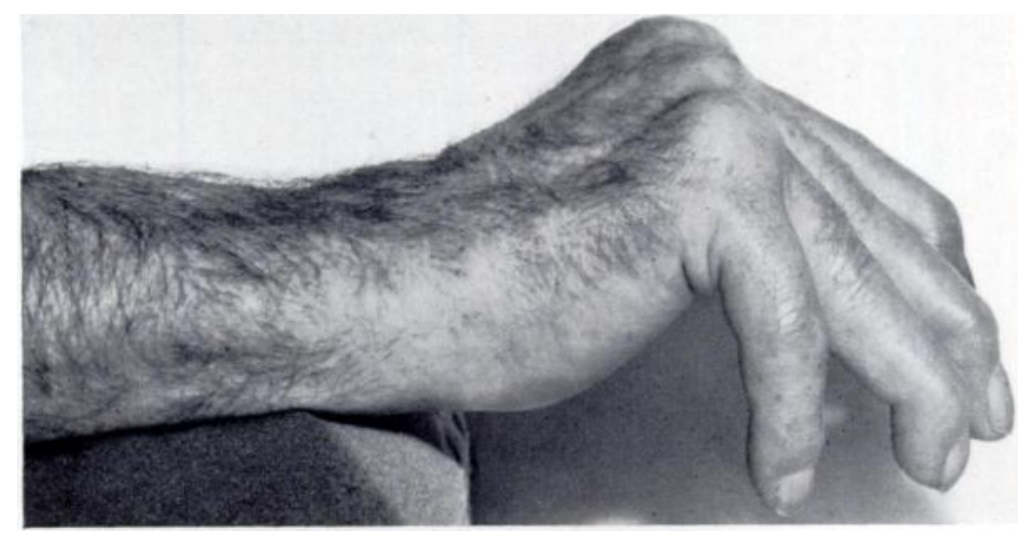

FIG. 1

Hand in rheumatoid arthritis showing flexion deformity at the metacarpophalangeal joints.

arthritis. Too often there has been a tendency to avoid operation simply because the patient has become adapted to his disability. Too often surgeons watch the deformity increase steadily throughout the time that something could have been done to prevent it, until in the end it is too late.

The normal alignment and posture of a joint, or complex of joints, depends on two kinds of stability: 1) stability depending on the intrinsic structure of the joint; and 2) stability depending on the balance of the extrinsic forces acting upon the joint, including gravity. 
Derangement of either of these forms of stability may cause secondary derangement of the other. Rheumatoid arthritis in the hand causes obvious disturbance of the intrinsic structure of joints, and we may therefore forget that in addition to arthritis there may also be osteitis, periostitis, myositis, neuritis, tenosynovitis, and even dermatitis as part of the rheumatoid process. We assume too easily that structural joint changes such as deformity, dislocation and ankylosis are primary, when in fact they are often secondary to an extrinsic imbalance. When it is remembered that tendon rupture disturbs function by an extreme form of muscle imbalance we are bound to look more critically at these deformities (Fig. 1). In this paper some examples of incomplete or complete interference with tendon action, and of deformity from extrinsic factors upsetting normal muscle balance, will be presented.

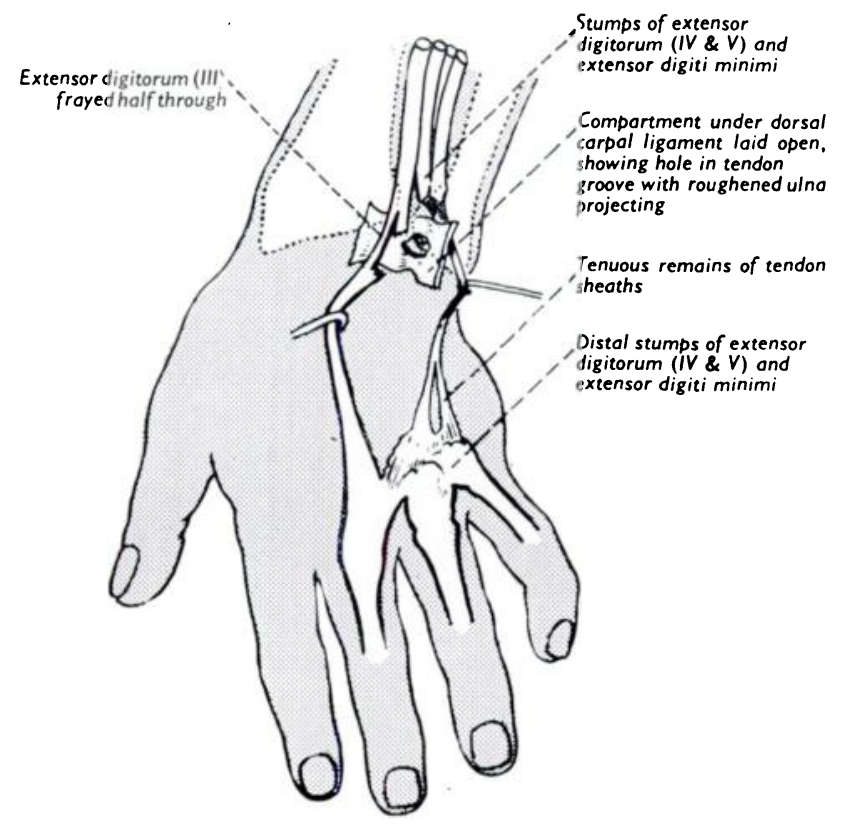

Fig. 2

Diagram of typical findings in attrition ruptures of extensor tendons.

\section{ATTRITION RUPTURES OF EXTENSOR TENDONS}

In 1948 two cases of sudden painless spontaneous rupture of the extensor communis tendons to the little and ring fingers from attrition on a rough bony spicule over an arthritic lower end of ulna were reported (Fig. 2) (Vaughan-Jackson 1948). No reference to such a rupture was found in the English literature of the previous thirty years. The cases were therefore reported as rarities, but it has now become clear that these ruptures are common. The original two cases were followed by another in which the findings at operation were predicted in every detail on the basis of the findings in the original two cases. Since then many further examples have been seen, and others have been reported elsewhere. I now have a personal series of thirty-five hands operated upon for this condition, as well as a number of others not treated by operation. Some patients declined operation; in others the associated deformity and disability in the hand were so great that it seemed unjustifiable to interfere, because the purpose of operation is primarily to stop the attrition of further tendons, and only secondarily to achieve a functioning repair (which is sometimes difficult).

It soon became apparent that those patients who noticed the sudden painless dropping of a finger at the metacarpo-phalangeal joint were matched by as many or more in whom 
rupture of one or more of the extensor tendons was found on clinical examination. It is among this group that fixed flexion deformities of the metacarpo-phalangeal joints were found, and in these subluxation or dislocation of the base of the permanently flexed proximal phalanx eventually occurs, setting the seal on the development and establishment of crippling deformity, and underlining the importance of recognising the lesion early while it is still correctible. Finally a number of cases of incomplete severance of the tendons by attrition have come to light at exploration of the wrist for other reasons (Fig. 14). It seems important therefore to describe in detail the manner in which the condition presents, the findings, and the results of treatment.

History - There may be no history of any recognisable incident at all. In this case the patient usually has fairly advanced rheumatoid arthritis, and it is not surprising that among all the other painful troubles sudden painless inability to extend a little finger passes unnoticed. Especially is this so if extension at the metacarpo-phalangeal joint is already hampered and partly restricted by a different kind of interference with free tendon action (to be considered later under ulnar deviation and the swan-neck deformity).

At the other extreme is the patient with mild "smouldering" disease, affecting perhaps only the wrist and inferior radio-ulnar joint, who is very little disabled. Such a patient will notice at once any sudden dropping of a finger and will seek advice.

The next feature of note is that the tendons rupture in succession starting from the ulnar side. In the little finger, which has two extensors, the slip from the extensor communis may rupture but the extensor proprius may escape, because it lies more medially and out of the way of the usual source of attrition. In such a case the first finger that is noticed to drop is the ring finger. After an interval of perhaps only a few days the middle finger drops and after a further interval perhaps the index will also be found affected. But the commonest order of casualties is little, ring, middle and index tendons. The index, being farthest from the danger spot, often escapes.

Occasionally the rupture of a tendon is accompanied by pain running up the forearm into the muscle belly, but it is seldom more than trivial. Sometimes there is warning of impending severance in the form of local pain felt only when the tendon is moved, but this seems to be commoner in tendons which run in tight canals, such as the extensor pollicis longus on the dorsum of the radius.

Clinical features - In the case of a recent rupture the first and the most striking feature is the loss of active extension at the metacarpo-phalangeal joint of the affected finger (Fig. 3). Passive extension is possible at first, but it becomes more and more restricted as time goes on and adaptive shortening takes place. On the dorsum of the hand the intact tendons can be seen and felt in normal tension. A severed tendon cannot be so distinguished (Fig. 4), and its distal end, retracted somewhat distally, may be seen standing out as a slight rounded lump in contrast to the flattened gap left by the much greater retraction of the proximal end. This lump can be rolled under the examining finger and pushed from side to side. Sometimes a ruptured tendon may appear to be intact, though much thinner and less taut than its intact neighbours. This appearance is due to the ends of the tendon having retracted within a sleeve of paratenon which collapses in the gap between the ends and often persists as a thin cord. This cord may even transmit a feeble extensor pull and so may mislead the surgeon from the true state of affairs.

The lower end of the ulna can often be felt to be hard and craggy, and the patient suffers pain in the inferior radio-ulnar joint on pronation and supination, the range of which is often restricted. Irrespective of the degree of involvement of the bones of the wrist and hand, a radiograph always shows bony involvement of the lower end of the ulna, and often there is much disorganisation of the inferior radio-ulnar joint (Fig. 5).

In long-standing cases with fixed flexion deformity of the metacarpo-phalangeal joints the dorsum of the hand is commonly thin and wasted even in the presence of marked synovial 
thickening of the wrist, and the absence of tendons standing out in normal tension is usually easily made out.

Findings at operation-The operative findings are so constant and so typical that it is startling

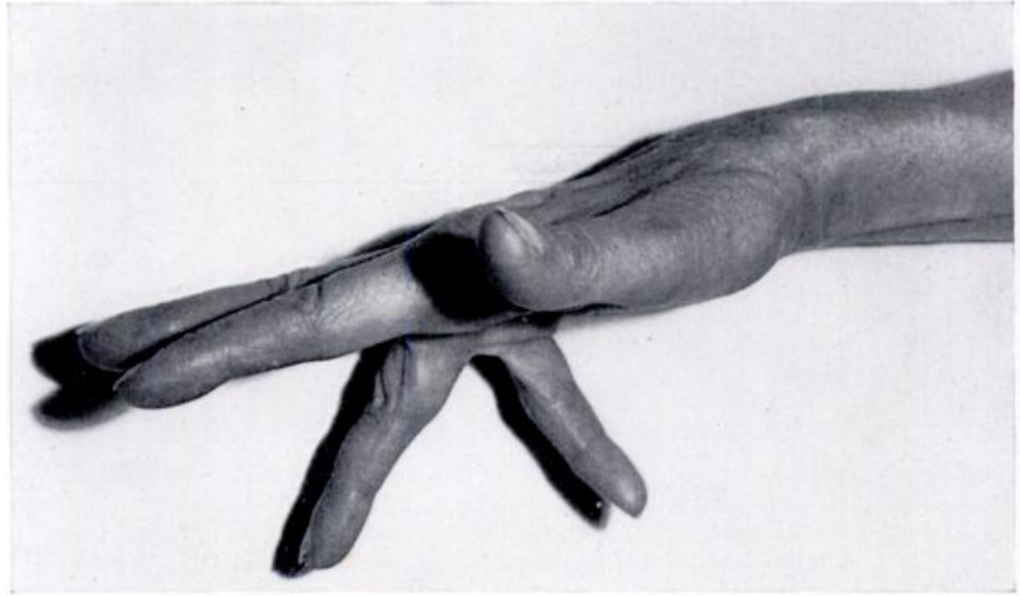

FiG. 3

Case 1-Attrition rupture of extensor communis digitorum tendons to ring and little fingers. Loss of active extension at the metacarpo-phalangeal joint.

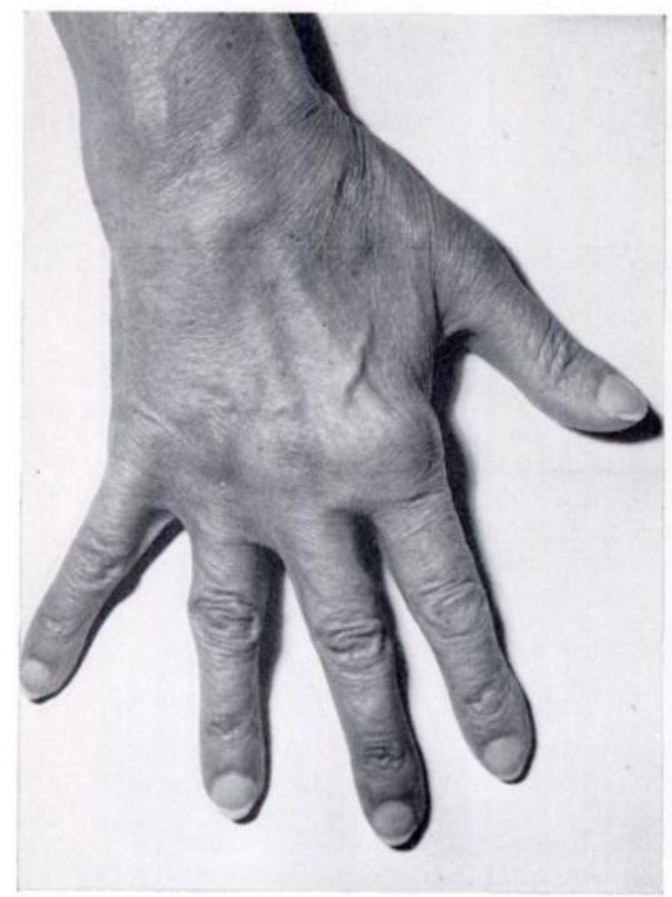

FIG. 4

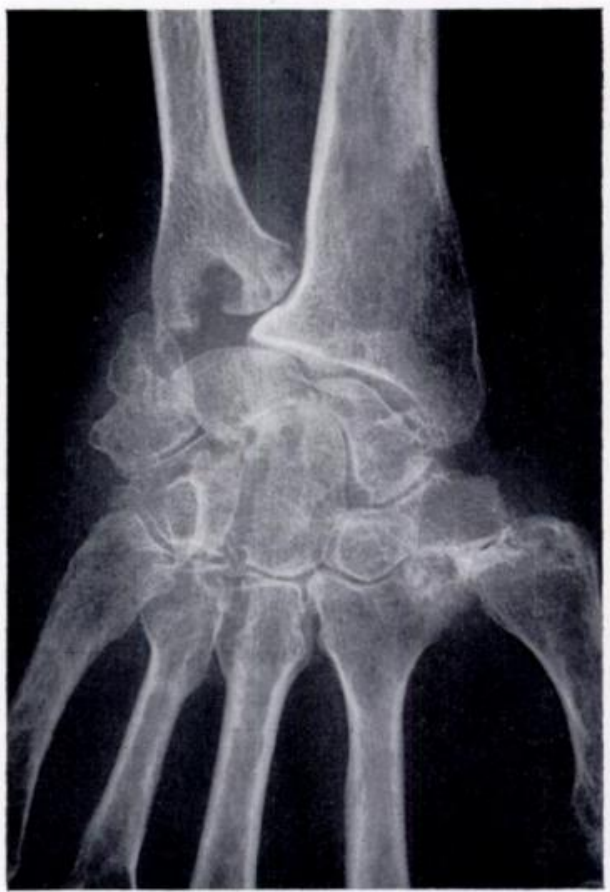

FIG. 5

Case 1. Figure 4-The intact extensor tendons to the index and middle fingers stand out in normal tension. Those to the ring and little fingers do not. Just distal to the wrist crease and on the ulnar side of the extensor digitorum to the middle finger can be seen the rounded prominence caused by the distal stumps of the tendons to the ring and little fingers. Figure 5-Radiograph of wrist showing marked involvement and erosion of lower end of ulna.

that there should be any doubt about the nature of the rupture. Nevertheless it has long been assumed that rupture took place spontaneously through an area of tendon weakened by the disease. It cannot be denied that this is a theoretical possibility, and it may be a true explanation

VOL. 44 B, NO. 4, NOVEMBER 1962 


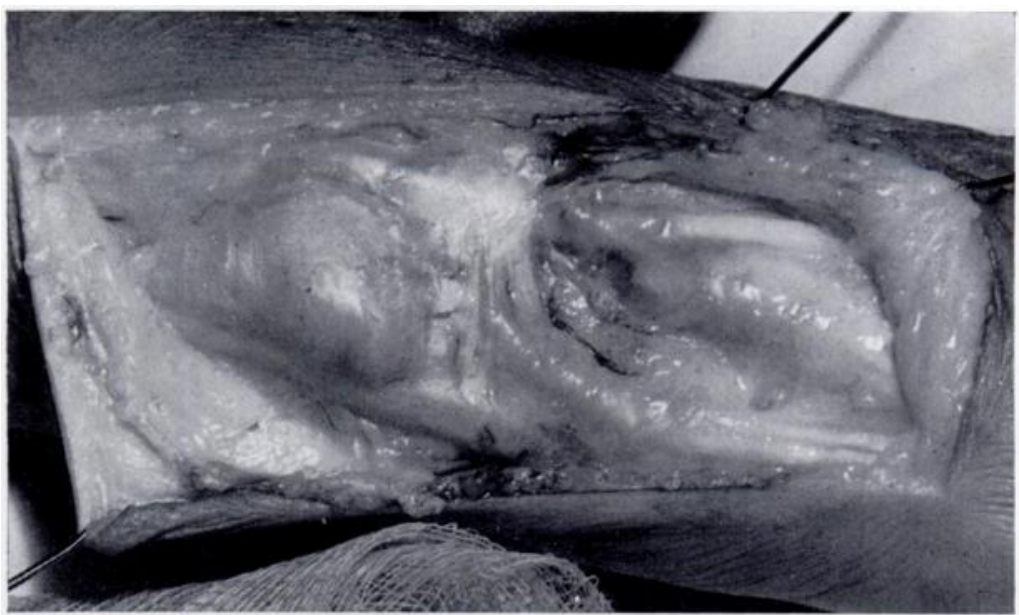

FIG. 6

Case 1-The dorsum of the wrist exposed. The lump formed by the distal stumps of the extensor tendons to the ring and little fingers is clearly seen on the right with the intact extensors to middle and index fingers beyond.

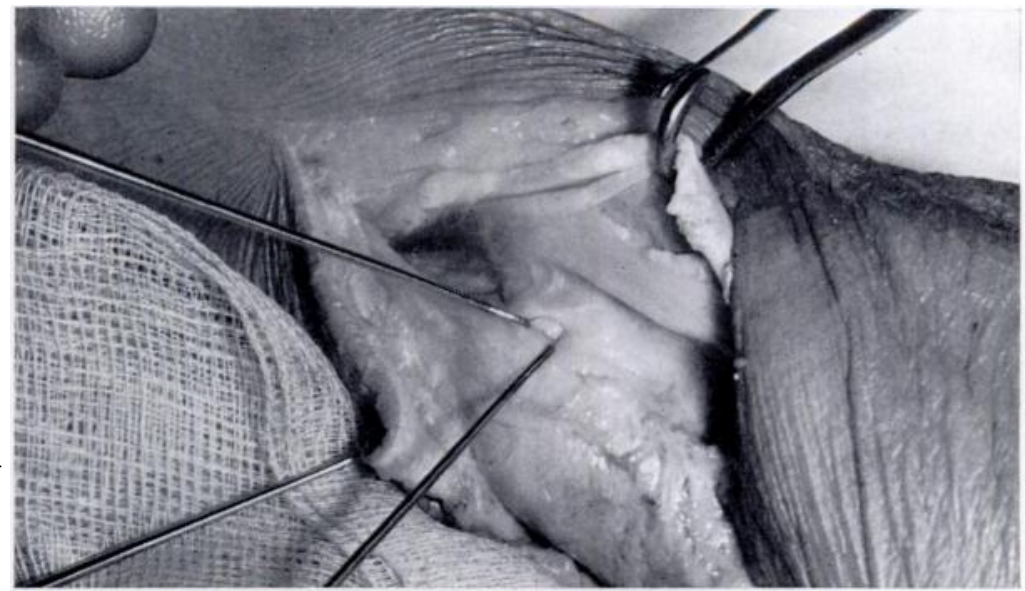

Fig. 7

Case 1-The hole in the floor of the tendon compartment is held open by two skin hooks (left). The abraded margin of the extensor tendon to the ring finger is seen in the grip of the button-hook retractor.

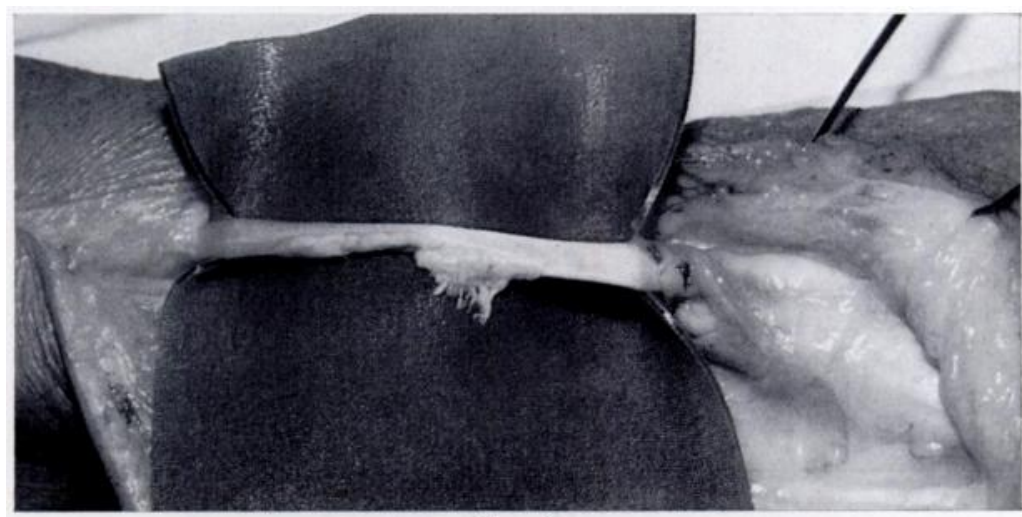

FIG. 8

Case 1-Typical appearance of a fresh abrasion of the ulnar margin of the next intact tendon. Note that there is no sign of any rheumatoid granuloma.

THE JOURNAL OF BONE AND JOINT SURGERY 
when the rheumatoid process is assisted by friction and constriction of the tendon in a tight fibro-osseous canal (as in the case of the extensor pollicis longus at the wrist). Rupture by attrition on a rough patch of bare bone has been found in these tunnels, but so also has spontaneous rupture with no evident cause of attrition. However, in the case of the common extensors rupturing at the level of the inferior radio-ulnar joint the significant fact is that in twenty-five consecutive cases the abrasion of the tendons and the cause of the attrition were manifest.

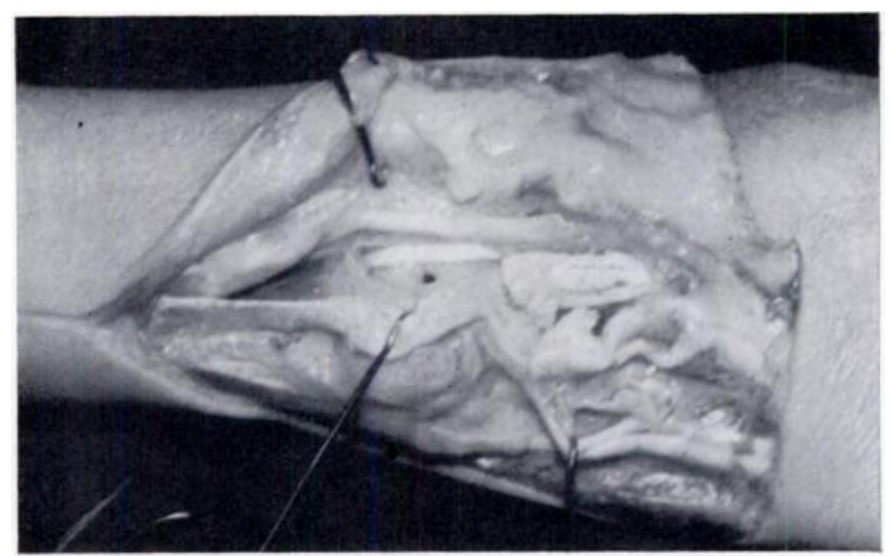

FIG. 9

Case 2-The dark dot is the hole in the floor of the tendon compartment. The ruptured tendon ends lie to the right; the next intact tendon has moved into position ready to be attacked.

It has to be realised that no positive conclusions can be drawn from the appearance of these ruptured tendons if they are explored late. The rheumatoid granuloma can be, in part at least, a reaction of the local tissue to injury. To find such a typical granuloma in a tendon end some months after the trauma of a rupture is not to be interpreted as evidence that it was there at the time of the rupture. The consistent failure to find any such granuloma in tendons explored within a day or two of the rupture, and the consistent finding of an obvious cause of attrition, demand the recognition of the true pathology, however much it may be true that spontaneous rupture through a granuloma can sometimes occur. Finally the finding under the microscope of bone spicules embedded in a tendon end free of rheumatoid granulations seems to decide the matter.

In a typical case a rough spicule on the lower end of the ulna can be seen and felt through an obvious hole in the floor of the dorsal tendon compartment when this is opened (Fig. 7). Over and over again the next remaining intact tendon has been seen not only lying close over this hole but displaying abrasion of its ulnar border in relation to the hole, the length of this abrasion corresponding exactly with the excursion of the tendon (Fig. 8). It seems clear that the mechanism is as follows: the rough lower end of the ulna corresponds to the nether millstone, the tendon over it to the upper millstone and the tissues of the floor of the tendon compartment are the grist, separating the two. Once the floor of the compartment is ground through and perforated the spicule of ulna attacks the overlying tendon. Its movement in pronation and supination saws across the line of the tendon and we know that a second rupture may follow the first after an interval of perhaps only a day or two. Once a tendon is parted its ends retract and the next intact one falls into place and is attacked in turn; and so the serial ruptures of tendon after tendon are produced (Fig. 9).

Sometimes one can find obvious recent abrasion of the next intact tendon at exploration soon after a rupture, and yet the cause of attrition may not be at all obvious. The spicule on the ulna has been found on many occasions to be hidden in two ways. The commonest 


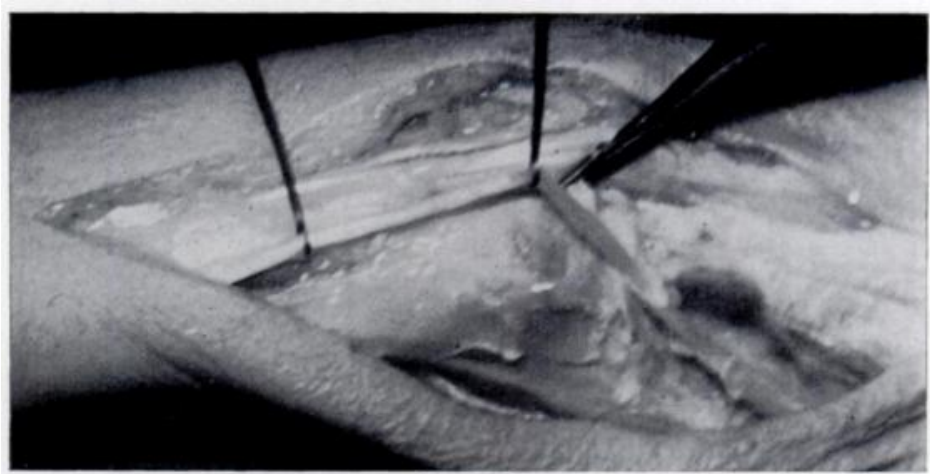

FIG. 10

Case 3-The extensor proprius tendon to the little finger has been lifted off the prominence made by the lower end of the ulna. On the top of this prominence thickened synovial tissue from the inferior radio-ulnar joint pouts through the hole in the capsule, obscuring it. The spicule on the ulna could be found with the probe within this pouting tissue.

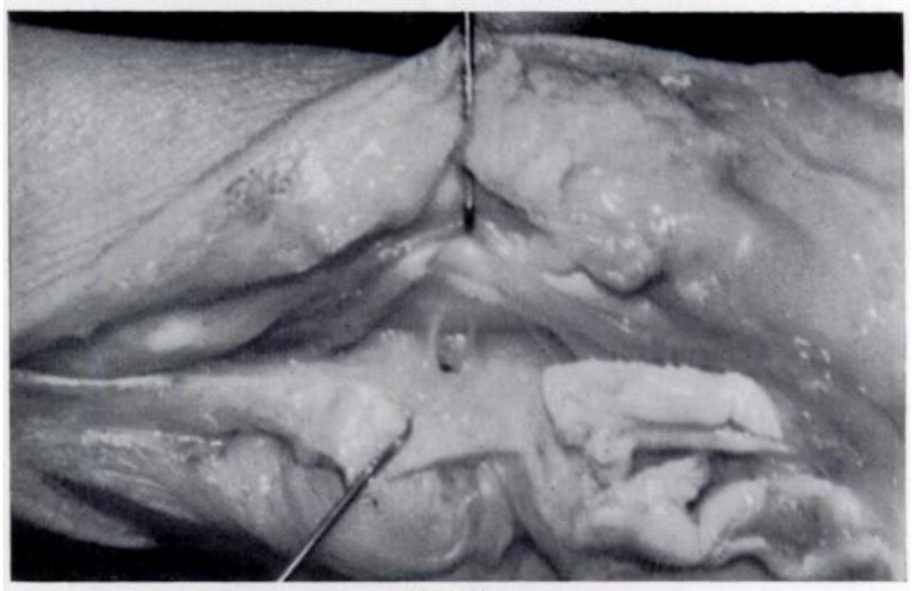

FIG. 11

Case 2-Note the valvular arrangement of tissue planes to be seen in the central hole. Ruptured tendon ends to the right. Note the translucent collapsed tube of paratenon in the grip of the upper hook. Follow it to the left where it is distended with fluid and the end of a ruptured tendon can be seen within it.

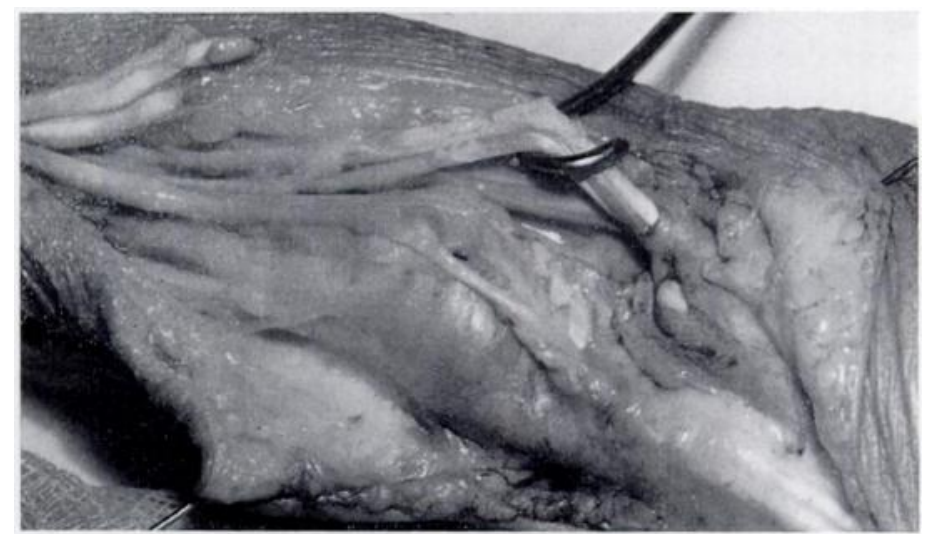

Fig. 12

Case 1-The dark hole over the lower end of the ulna is seen centrally. A strand resembling stretched and thinned-out tendon lies beside it. Follow this to the leftwhere it is seen to spread out over a bulbous ruptured tendon end similar to andat the same level as the "naked" rounded tendon stumps above it. 
finding is that a mass of rheumatoid synovial tissue from within the inferior radio-ulnar joint pouts through the hole and hides it but does not prevent attrition proceeding (Fig. 10). In such a case the hole can be found with a probe.

In other cases the perforations in the layers of tissue in the floor of the tendon compartment are only " in register" at one point in the pronation-supination range. At operation, if the wrist is passively pronated and supinated, the hole in the synovial layer of the dorsal compartment can be seen to move in relation to the hole in the fibrous layer under it, and both are seen to move in relation to the hole in the synovial lining of the inferior radio-ulnar joint. This valvular arrangement closes the hole throughout all but a small part of the pronationsupination range (Fig. 11).

These findings are present at exploration soon after a rupture. At late exploration there may be no spicule nor hole apparent, and one may surmise that once several of the tendons have parted and retracted there is no longer an upper millstone and the hole may have healed. In this way a true attrition rupture may be unable to be recognised.

A further typical feature is the strand of tissue that unites the severed ends of many of these tendons. This has been described in the past as a "stretched degenerate rheumatoid tendon" and admittedly this thin white opaque strand looks remarkably like a stretched tendon. The first finding that threw doubt on this explanation was that if this strand is dissected out it can always be shown to reach and spread out over the surface of the severed tendon ends which are quite distinct, rounded, opaque, and yellowish within it (Fig. 12). Next, under the microscope the strand is not composed of tendon fibres, but of fibrous tissue with synovial elements. Lastly, early exploration of these ruptured tendons demonstrates the true state of affairs unmistakably. A week or ten days after the rupture the strand will look semi-translucent and bluish, not at all like the apparently fibrous strand seen at a later stage. Earlier still it will look translucent as though it contains fluid (Fig. 11), which in fact it may, for at exploration within a day or so of the rupture the strand can be seen with certainty to be the collapsed tube of paratenon within which the tendon ends have separated, and this tube contains a little synovial fluid. Moreover the tendon ends are demonstrably within it (Fig. 15). Treatment-Resection of about an inch of the lower end of the ulna removes the cause of attrition and "stops the rot" (Fig. 13). It is a procedure with hardly any penalties. It is said that patients will complain of painful clicking of tendons over the end of the ulnar shaft after such a resection, but I have never encountered any such trouble.

If the radio-carpal joint is much damaged by the disease, removal of the support provided by the lower end of the ulna may allow the carpus to slide medially on the lower end of the radius even to the point where a radiocarpal arthrodesis may be necessary, but in most such cases an arthrodesis would have been necessary in any event.

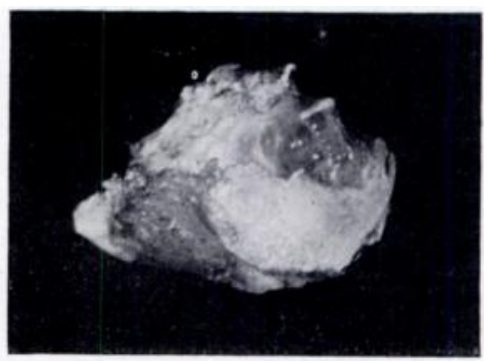

FIG. 13

Case 2-Excised lower end of ulna seen end on. The uppermost spicule had done the damage.

On the other hand the operation has additional advantages and should be performed far more often than it is, simply for the relief of painful restriction of pronation and supination, in which respect it is uniformly successful.

Repair of the ruptured tendons is necessary not only to restore normal function but also, particularly, to get the metacarpo-phalangeal joints working again in their middle range. If left in extreme flexion they are under unnatural strain. They will first become fixed in this position; next the rheumatoid process will attack them with avidity, the greater for their being at a disadvantage; lastly they will become disorganised and dislocated by the unbalanced pulls acting on them.

VOL. 44 B, NO. 4, NOVEMBER 1962 
The "rheumatoid battlefield" on which these repairs have to be undertaken may be daunting, with its masses of bulging unhealthy synovial tissue, but nevertheless the tendons themselves usually look surprisingly normal by comparison with their surroundings, and results are not disappointing (Fig. 14). Almost any kind of tendon repair can work in this situation, and even fairly rough " cobbling" together of tendons has been successful. The most promising cases are those in which not more than two tendons have been severed.

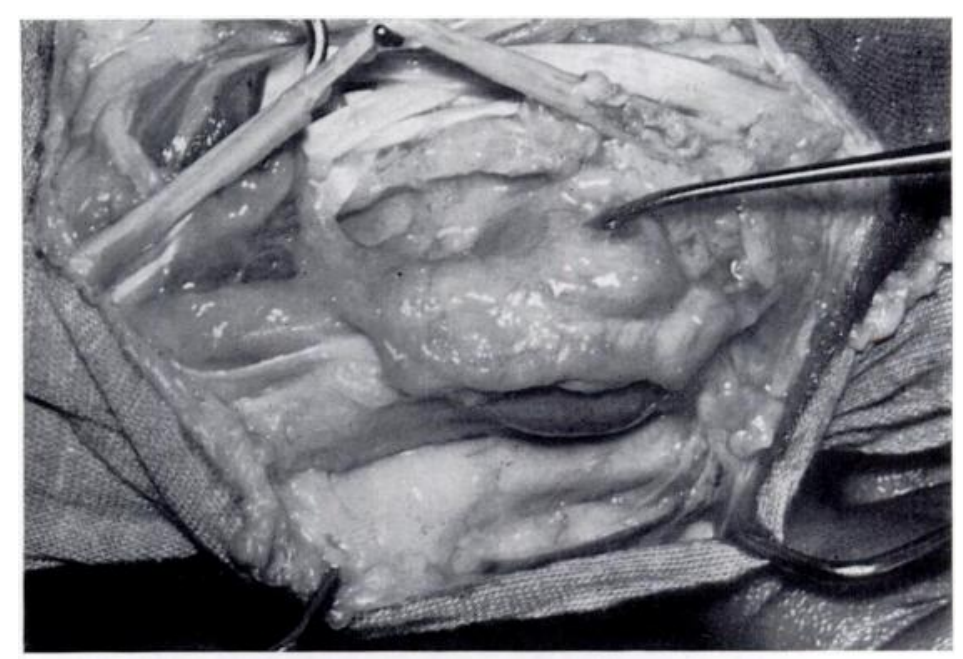

FIG. 14

Case 4-Despite the obvious masses of rheumatoid synovial tissue the tendons appear normal. The end of the probe indicates the hole over the ulna and immediately above it early abrasion is apparent on the ulnar margin of the tendon lifted on the hook. This attrition was discovered incidentally when the wrist was exposed for a radio-carpal arthrodesis.

It is then possible to separate a longitudinal sliver from the margin of the next intact tendon and swing its distal end across to the distal stump of the severed tendon. Only one suture line per tendon is necessary and the line of approach of the transferred tendon is satisfactory. If several distal stumps are attached to one " motor" they diverge too sharply from its line of action and the pull to the outermost stumps is at an angle and very inefficient.

Finally, unhealthy as the surrounding rheumatoid synovial tissue may be, it is all that one has for covering suture lines to prevent them from adhering to skin, and for this reason one must "spring-clean" the area with discretion. Adhesions may occur in any case, and limit the excursion of the repaired extensors; when this happens tenolysis is often successful.

The state of activity of the rheumatoid process seems to have little or no bearing on the indications for operation except in those cases of florid disease under heavy steroid therapy, when the wisdom of carrying out any kind of surgical procedure will be in doubt. Apart from this the need to arrest the series of ruptures overrides other conditions, and the activity of the disease does not appear to affect healing of transferred tendons or grafts.

\section{OTHER ATTRITION RUPTURES}

Flexor tendons-Other varieties of attrition rupture are coming steadily to light, the latest being in the foot. In the hand, rupture of the flexor tendons by attrition in the carpal tunnel is only slightly less common than rupture of the extensors. The usual cause of attrition is an area of cortical erosion on the palmar surface of one of the carpal bones. Rheumatoid arthritis is by far the commonest cause, and the erosion of cortex exposes a "glass paper" surface of cancellous bone. Every bone in the carpus has been implicated, the hardest lesion 
to find having been one tucked away under the hook of the hamate bone. In only one case was the cause of attrition on the lower end of the ulna, and in that case the rupture was a typical transverse pronation-supination "cross-cut" attrition. In all the others the abrasion was in the line of travel of the tendons, resulting in a longitudinal "combing out" of the tendon fibres until sometimes the cylindrical body of the tendon was transformed into a sheet of separated but intact groups of fibres.

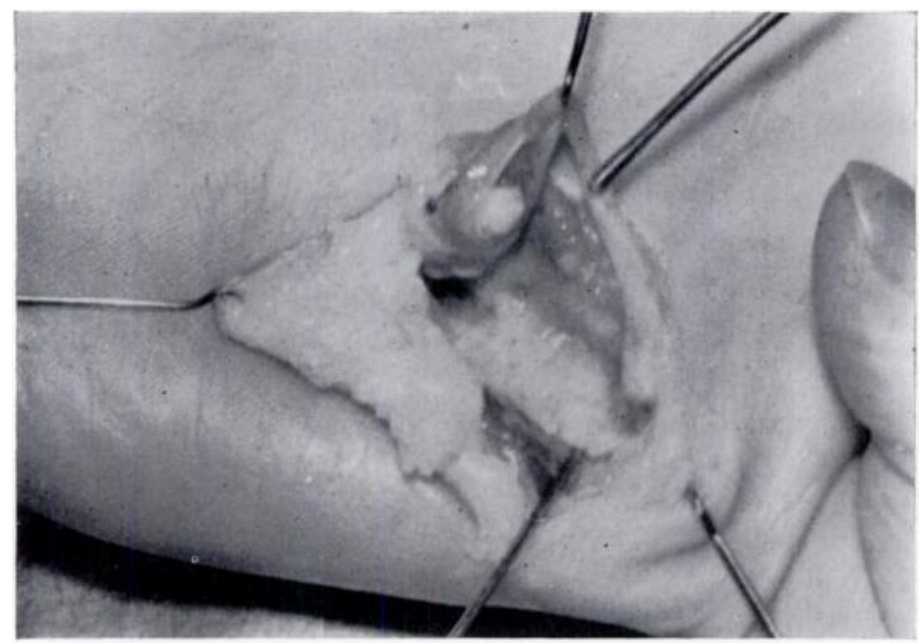

FIG. 15

Case 5-Attrition rupture of both flexors in the little finger on a roughened sesamoid bone. The lower probe indicates the sesamoid. The proximal tendon stump peeps out of the tube of paratenon. The upper probe indicates the distal end of sublimis within this tube. The distal end of profundus peeps out from the right hand margin of the wound, where the tube of paratenon can be seen leaving the margin of sublimis to embrace the stump of profundus.

In every case, by the time the tendons were explored all the tendons in the carpal tunnel were damaged and tattered, an observation that underlines the fact that the tendons do not maintain their text-book relationships except when at rest. During activity they slide and move, deep ones becoming superficial and vice versa, according to which is in active tension. In this way a small lesion can damage all of them. It is evident, too, that the process of attrition is painless, or else its discomforts are obscured by the familiar twinges of the rheumatoid process itself. That it can be painless is also demonstrated by cases of attrition after old injury, as for instance after a Colles's fracture resulting in a spicule of bone projecting into the carpal tunnel.

With this pathological picture in mind it is not surprising that many of these patients display, clinically, evidence of far more widespread damage than their presenting symptoms would lead one to expect. The presenting symptom is nearly always inability to flex a terminal interphalangeal joint. It is by no means uncommon to find that several or even all the flexor sublimis tendons are ruptured as well. So long as the flexor profundus is intact the patient does not notice the loss of a sublimis.

It therefore behoves the surgeon to move fast under such circumstances. Tendon repairs are difficult. Free grafts adhere readily to all the damaged tendons, and the best hope lies in removing the cause and attaching the severed profundus to its nearest intact, if damaged, neighbour.

In removing the cause, usually all one can do is to excavate and countersink the bare bone well below the surface. There is little or no soft tissue with which to cover it, and its 
dead white appearance leaves one with doubts whether it will be satisfactorily covered spontaneously.

Two kinds of deformity are likely to result from this sort of tendon damage. The first is the hyperextended terminal interphalangeal joint which cannot be flexed. This occurs most notably in the thumb. Secondly, if the flexor sublimis is ruptured the flexor-extensor balance of the proximal interphalangeal joint is lost and it tends rapidly to become hyperextended,

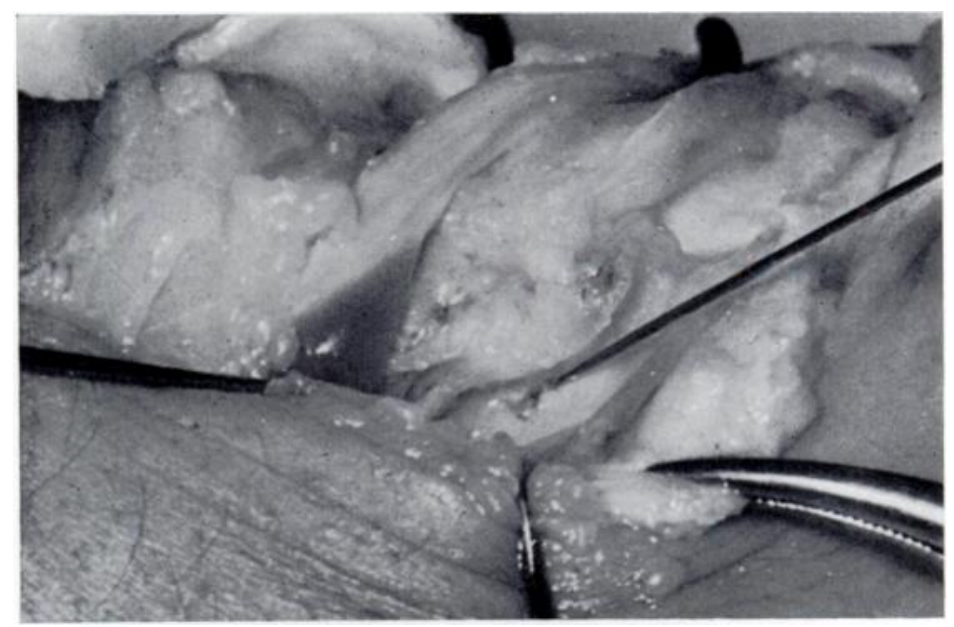

Fig. 16

Case 6-The probe indicates the rough area in the floor of the groove for extensor pollicis longus, which has been displaced out of it and lies in the grip of the large hook.

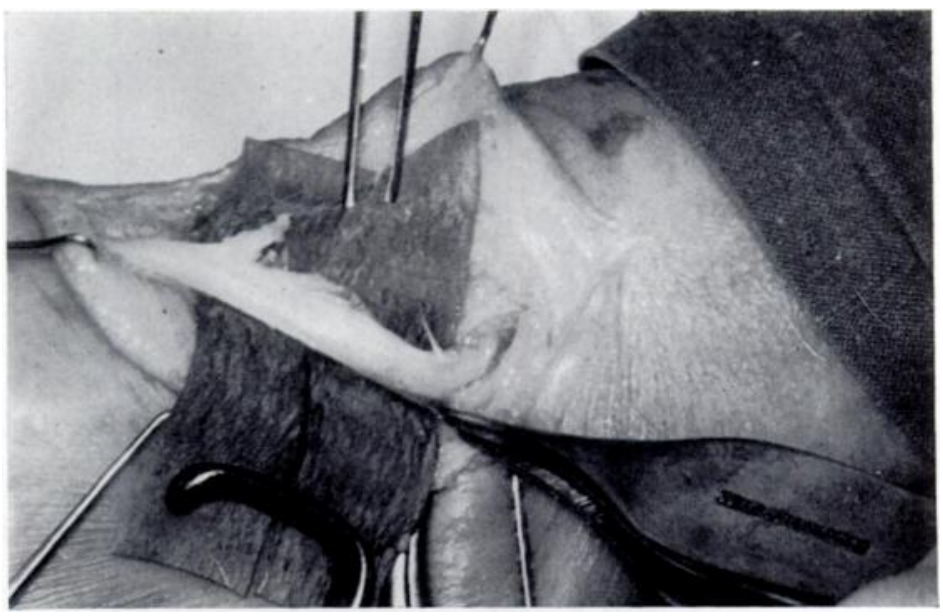

Fig. 17

Case 6-This shows the attrition on the margin of extensor pollicis longus.

and the finger soon assumes the classical " swan-neck" deformity. With scarcely less speed the rheumatoid process fixes the deformity and the finger becomes stiff in the position of least useful function.

Attrition ruptures of flexor tendons in fibro-osseous tunnels-These are less common. One case of attrition of both flexor tendons on a roughened sesamoid bone at the base of the little finger was doubtless a rarity (Fig. 15). Attrition ruptures, however, have been seen in the digital sheaths, in the flexor pollicis longus in its sheath, and in the extensor pollicis longus 
on the dorsum of the radius, but never in the tendons at the radial styloid. More often no cause of attrition can be found, and one may surmise that perhaps friction and degenerative changes are more often to blame. None the less, ruptures of these tendons, from whatever cause, are fairly common in patients with rheumatoid arthritis. Loss of the flexor pollicis longus, for example, carries a considerable penalty in the shape of clumsiness in fine movements (as in doing up a button, winding a watch or sewing), and too often the reason for the hyperextended terminal phalanx is not understood. Ruptures of these tendons do tend to give some warning in the form of pain on movement of the tendon, and in one case the extensor pollicis longus was explored because of this and found half severed on the usual piece of "glass paper" bone in its groove (Figs. 16 and 17). The cause of attrition was removed by countersinking. No subsequent rupture of the tendon occurred.

\section{SUMMARY}

1. The posture of deformed finger joints in rheumatoid arthritis needs close analysis in terms of disturbed muscle balance. Although disorganisation of the joint itself may be the primary factor in the development of deformity, the deformity is often secondary to an extrinsic disturbance of muscle balance.

2. The part played by tendon ruptures in producing such imbalance is discussed.

I would like to record my special indebtedness to $\mathrm{Dr}$ W. S. Tegner and $\mathrm{Dr}$ R. M. iMason at the London Hospital, and Dr R. A. Moir at St Bartholomew`s Hospital, Rochester, for their great interest and endless cooperation in the study of these abnormalities, and to Mr R. F. Ruddick for the great skill and care that he has put into producing the photographs.

\section{REFERENCES}

Brewerton, D. A. (1957): Hand Deformities in Rheumatoid Disease. Annals of the Rheumatic Diseases, $16,183$.

Ehrlich, G. E., Peterson, L. T., Sokoloff, L., and Bunim, J. J. (1959): Pathogenesis of Rupture of Extensor Tendons at the Wrist in Rheumatoid Arthritis. Arthritis and Rheumatism, 2, 332.

JAMES, J. I. P. (1949): A Case of Rupture of Flexor Tendons Secondary to Kienböck's Disease. Journal of Bone and Joint Surgery, 31-B, 521.

Kaplan, E. B. (1953): Functional and Surgical Anatomy of the Hand. Philadelphia and London: J. B. Lippincott Company.

Laine, V. A. I., Sairanen, E., and Vainio, K. (1957): Finger Deformities caused by Rheumatoid Arthritis. Journal of Bone and Joint Surgery, 39-A, 527.

Straub, L. R., and Wilson, E. H., Jun. (1956): Spontaneous Rupture of Extensor Tendons in the Hand Associated with Rheumatoid Arthritis. Journal of Bone and Joint Surgery, 38-A, 1,208.

VAughan-Jackson, O. J. (1948): Rupture of Extensor Tendons by Attrition at the Inferior Radio-ulnar Joint. Journal of Bone and Joint Surgery, 30-B, 528.

Vaughan-Jackson, O. J. (1959): Attrition Ruptures of Tendons as a Factor in the Production of Deformities in the Rheumatoid Hand. Proceedings of the Royal Societyof Medicine (Section of Orthopaedics), 52, 132. 